colorectal screening, there remains a need to identify which system has the greatest diagnostic accuracy. Therefore, we prospectively compared three existing CRC risk score systems in their ability to predict advanced colorectal neoplasia in Australian population; the Asia-Pacific Colorectal Screening (APCS) score; Hong Kong Score (2014); and Imperiale Score (2015).

Methods Patients scheduled for colonoscopy assessment, both with or without gastrointestinal symptoms, were recruited. FOBT positive patients were included, but those who had an examination of the colon, including colonoscopy, within the last five years were excluded. Univariate and multivariate logistic regression was applied to identify significant risk factors for advanced neoplasia. For each patient, the $\underline{3}$ different risk scores were applied and the performance of each score in the prediction of advanced neoplasia was compared by examining the area under the curve (AUC) value.

Results A total of 361 patients undergoing colonoscopy $(48.2 \%$ male, median age 60 years) were prospectively recruited. The prevalence of adenomas was $31.6 \%$, and $10.0 \%$ for advanced adenoma including 8 CRC (2.2\%). Upon multivariate analysis, age and male sex were found to be significant risk factors $(\mathrm{P}=0.001, \mathrm{P}=0.002)$. For predicting the prevalence of advanced neoplasia, the APCS score had AUC 0.71 (95\%CI 0.63-0.79), Hong Kong Score 0.69 (95\%CI 0.61-0.78), and Imperiale Score 0.68 (95\%CI 0.59-0.77). Using a non-parametric comparison of the AUCs, there was no statistical significance between each of the scores for both symptomatic and asymptomatic populations $(\mathrm{P}=0.37$ for APCS vs Hong Kong Score; $\mathrm{P}=0.32$ for APCS vs Imperiale Score; $\mathrm{P}=0.43$ for Hong Kong Score vs Imperiale Score).

Conclusions All three scores are equally effective in stratifying the population into low and high risk colorectal neoplasia groups, and may be used to prioritise patients for colorectal screening.

\section{IDDF2019-ABS-0132 METACHRONOUS GASTRIC CANCER PREVENTION WITH ASPIRIN AFTER ENDOSCOPIC SUBMUCOSAL DISSECTION FOR EARLY GASTRIC CANCER: PROTOCOL OF A MULTI-CENTER RANDOMIZED CONTROLLED TRIAL}

${ }^{1}$ Sho Suzuki*, ${ }^{2}$ Siew Ng, ${ }^{3}$ Takahisa Furuta, ${ }^{1}$ Takuji Gotoda, ${ }^{4}$ Kazuma Fujimoto, ${ }^{2}$ Francis KL Chan. 'Nihon University School of Medicine, Japan: ${ }^{2}$ The Chinese University of Hong Kong, Hong Kong; ${ }^{3}$ Hamamatsu University School of Medicine, Japan; ${ }^{4}$ Saga University, Japan

\subsection{6/gutjinl-2019-IDDFabstracts.7}

Background Epidemiological studies suggest that long term low-dose aspirin use reduces the risk of gastric cancer. However, no randomized control studies have been conducted to assess the role of aspirin in the reduction of occurrence of gastric cancer. This study aims to investigate the chemo-preventive effect of aspirin on the development of metachronous gastric cancer after endoscopic submucosal dissection (ESD) for early gastric cancer (EGC).

Methods A total of 1120 patients received ESD for EGC and Helicobacter pylori eradication at 33 institutions among Japan and Hong Kong will be enrolled in this study. Enrolled patients will be randomly assigned to either receive aspirin 81 mg once daily or nothing for 5 years at a 1:1 allocation ratio.
Patients will be examined endoscopically for metachronous gastric cancer at 12, 24, 36, 48 and 60 months after randomization. The primary outcome measure is the development of metachronous gastric cancer at 5 years. Metachronous gastric cancers will be confirmed through histological examination of samples obtained through biopsy, or endoscopic or surgical resection. A blinded adjudication panel consisting of three pathologists will diagnose all specimens. The histological criteria of gastric cancer will be defined as categories 4 and 5 with the Vienna classification.

Results This study was approved by Institutional Review Board of Nippon Medical School Foundation on February 25, 2019. The start of the recruitment is planned in April 2019 and will continue for a 3-year period.

Conclusions The findings of this study will be submitted to a peer-reviewed journal. Abstracts will be submitted to relevant national and international conferences. This study is registered with the University Hospital Medical Information Network (UMIN) Clinical Trials Registry (www.umin.ac.jp/ctr/; identification No.: UMIN000031302).

\section{IDDF2019-ABS-0142 COMBINATION OF ERCP AND EUS-GUIDED BILIARY DRAINAGE (CERES) VERSUS PTBD FOR MALIGNANT HILAR BILIARY OBSTRUCTION: A MULTICENTER PROSPECTIVE COMPARATIVE COHORT STUDY (THE CERES STUDY)} ${ }^{1}$ Passkorn Sodarat, ${ }^{1}$ Orathai Seabmuangsai, ${ }^{3}$ Rommel Romano, ${ }^{3}$ Sureeporn Jangsirikul, ${ }^{3}$ Wiriyaporn Ridtitid, ${ }^{3}$ Phonthep Angsuwatcharakon, ${ }^{3}$ Thawee Ratanachu-ek, ${ }^{3}$ Rungsun Rerknimitr. ${ }^{1}$ Roi-Et Hospital, Ministry of Public Health, Thailand; ${ }^{2}$ ThaBo Crown Prince Hospital, Ministry of Public Health, Thailand; ${ }^{3}$ Gastrointestinal Endoscopy Excellence Center and Division of Gastroenterology, Department of Medicine, Faculty of Medicine, Chulalongkorn University and King Chulalongkorn Memorial Hospital, Thai Red Cross Society, Bangkok, Thailand

\subsection{6/gutjnl-2019-IDDFabstracts.8}

Background Combination of ERCP and EUS-BD (CERES) including EUS-guided hepaticogastrostomy (EUS-HGS) and EUS-guided hepaticoduodenostomy (EUS-HDS) may provide complete $\mathrm{BD}$ in bismuth 3 and 4 malignant hilar biliary obstruction (MHBO. This study aims to prospectively compare the efficacy of CERES versus PTBD in MHBO.

Methods Patients with MHBO were recruited. Patients presented to endoscopy and interventional radiology service received CERES (group A) and PTBD (group B) as primary BD method, respectively. Technical and clinical success rate (TSR and CSR) and time to RBO (T-RBO = time from last successful $\mathrm{BD}$ to biliary reintervention procedure) were recorded. The study was conducted during March 2016 until October 2018 as a multicenter study of 3 Thai hospitals (King Chulalongkorn Memorial Hospital of Thai Red Cross Society, Tha-Bor Hospital, and Roi-Et Hospital) under the Thai Association for Gastrointestinal Endoscopy (TAGE) guidance.

Results 45 patients $(23 \mathrm{M}, 22 \mathrm{~F})$ were recruited into group A $(n=23)$ and group $B(n=22)$. One patient from group A was withdrawn due to loss to follow up. Demographic data and results of both groups are shown in table 1 . Overall TSR, CSR, and complication rate (CR) of group A versus B were $90.9 \%(20 / 22)$ vs $100 \%(22 / 22) \quad(p=$ not significant (NS)), $81.8 \%(18 / 22)$ vs $86.4(19 / 22) \quad(\mathrm{p}=\mathrm{NS})$, and $18.2(4 / 22)$ vs 